\title{
Is Heteropolymer Freezing Well Described by the Random Energy Model?
}

\author{
Vijay S. Pande†, Alexander Yu. Grosberg †‡, Chris Joerg§, Toyoichi Tanaka† \\ $\dagger$ Department of Physics and Center for Materials Science and Engineering, \\ Massachusetts Institute of Technology, Cambridge, Massachusetts 02139, USA \\ $\ddagger$ On leave from: Institute of Chemical Physics, Russian Academy of Sciences, Moscow 117977, Russia \\ $\S$ Laboratory for Computer Science, Massachusetts Institute of Technology, Cambridge, Massachusetts 02139, USA
}

\begin{abstract}
It is widely held that the Random Energy Model (REM) describes the freezing transition of a variety of types of heteropolymers. We demonstrate that the hallmark property of REM, statistical independence of the energies of states over disorder, is violated in different ways for models commonly employed in heteropolymer freezing studies. The implications for proteins are also discussed.
\end{abstract}

\section{INTRODUCTION}

Heteropolymer freezing is widely recognized as a model for protein folding. By heteropolymer freezing, we refer to the transition from a phase in which many conformations $\left(\mathcal{O}\left(e^{N}\right)\right)$ dominate equilibrium to one in which only one or very few $(\mathcal{O}(1))$ are thermodynamically relevant. Remarkable progress in the understanding of heteropolymer freezing has been achieved in recent years mainly due to the concepts borrowed from the statistical mechanics of spin glasses, such as the so-called Random Energy Model (REM) first suggested by Derrida [1].

Noting the properties of REM - a rugged free energy landscape and the statistical independence of states Bryngelson and Wolynes first proposed the use of REM for protein folding [2]. Other approaches, which employed the sophisticated machinery of replica mean field theory and began with an, although simplified, microscopic model of random heteropolymer chains, also led to REM-like conclusions [3].

However simple technically, REM and related ideas have proven to be extremely fruitful in protein folding studies [3 77]. It turned out so successful, that at present, it is often considered not as just a model, which may or may not be applicable under different circumstances, but as a simple and adequate universal language. While REM seems like technical theoretical jargon, it is closely related to the set of physical insights which is often used without mentioning (and perhaps without considering) any theoretical concepts at all (eg. Ref. [9]).

The validity of REM was proven [3, 10] for maximally compact heteropolymers with a Gaussian distribution of monomer interaction energies. On the other hand, there are many observations that are hardly understandable using REM |11]. Thus, the goal of the present work is to scrutinize the real strength and validity of REM in the context of heteropolymer freezing and protein folding studies. We will show that REM is indeed often applicable, but it is far from being applicable universally; its applicability is a highly non-trivial property, and it fails or is at least questionable in many cases.

\section{QUALITATIVE CONSIDERATIONS}

Heteropolymer freezing is said to be due to the frustrated interplay between three fundamental polymeric elements: sequence, set of conformations available for the given chain, and the nature of interaction between monomer species. More specifically, the freezing of a polymer in a certain conformation is governed by the competition of two conflicting factors. First, how well are the interactions adjusted in this conformation? Clearly, this depends on the character of interaction between monomers. A broad distribution of the types of monomer species favors energetically strong preferences between conformations. Second, how many conformations exist in the vicinity of a given conformation? This defines the entropy and depends entirely on the internal geometry of the space of available conformations (chain flexibility, topology, etc.).

REM specifies this picture of freezing by the assertion that for each sequence, the polymer energy $E_{\alpha}$ of conformation $\alpha$ is taken randomly from probability distribution over disorder $P(E)$, which is the same for all $\alpha$; furthermore, they are taken independently, such that joint probability is directly expressed in terms of $P(E)$ : $P\left(E_{\alpha}, E_{\beta}\right)=P\left(E_{\alpha}\right) P\left(E_{\beta}\right)$. Obviously, this cannot be valid exactly. Indeed, for conformation $\beta$ which is only a minor conformational rearrangement of $\alpha$, many contributions to the respective energies are identical and thus the energies are clearly correlated. REM validity requires that such pairs of conformations are rare.

The simplest quantitative measure of statistical dependence between the energies of two given conformations $\alpha$ and $\beta$ over the set of sequences can be obtained by taking correlation $\left\langle E_{\alpha} E_{\beta}\right\rangle-\left\langle E_{\alpha}\right\rangle\left\langle E_{\beta}\right\rangle=\left\langle\delta E_{\alpha} \delta E_{\beta}\right\rangle$, where $\langle\ldots\rangle$ denotes averaging over sequences. REM invalidity can be demonstrated by the non-vanishing of this correlator. We consider the Hamiltonian

$$
\mathcal{H}=\sum_{I \neq J} B\left(s_{I}, s_{J}\right) \delta\left(\mathbf{r}_{I}-\mathbf{r}_{J}\right)
$$

where $s_{I} \in\{1, \ldots, q\}$ is the species of monomer number 
$I, q$ is the number of species, $B_{i j}$ is the matrix of speciesspecies interactions, $\mathbf{r}_{I}$ is the position of monomer $I$, and $\delta\left(\mathbf{r}-\mathbf{r}^{\prime}\right)$ is the function that is concentrated on nearest neighboring space points. We implicitly assume here, that position vectors $\mathbf{r}_{I}$ are such that the conditions of chain connectivity (unitary spatial distance between sequential monomers), excluded volume and constant density (on the lattice, for instance, every site is occupied by one and only one monomer) are all met. This is the most general model for the case when heterogeneity comes exclusively from pairwise, short range interactions [8]. For the Hamiltonian (1), we find

$$
\left\langle\delta E_{\alpha} \delta E_{\beta}\right\rangle=\left\langle\delta B^{2}\right\rangle \mathcal{Q}_{\alpha, \beta}+N z^{2} \sum_{i j k} p_{i} \delta B_{i j} p_{j} \delta B_{j k} p_{k}
$$

where $p_{i}$ is the probability of finding a monomer of species $i, \delta B_{i j} \equiv B_{i j}-\sum_{k l} p_{k} B_{k l} p_{l},\left\langle\delta B^{2}\right\rangle \equiv$ $\sum_{i j} p_{i}\left(\delta B_{i j}\right)^{2} p_{j}$ is the variance of the elements of the interaction matrix, and $\mathcal{Q}_{\alpha \beta} \equiv \sum_{I \neq J} \delta\left(\mathbf{r}_{I}^{\alpha}-\mathbf{r}_{J}^{\alpha}\right) \delta\left(\mathbf{r}_{I}^{\beta}-\mathbf{r}_{J}^{\beta}\right)$ is the conventionally defined overlap between conformations [5, 10]. We have taken into account here the condition that the polymer is maximally compact, so that each monomer has $z$ space neighbors, and thus $\sum_{I \neq J \neq K} \delta\left(\mathbf{r}_{I}^{\alpha}-\mathbf{r}_{J}^{\alpha}\right) \delta\left(\mathbf{r}_{J}^{\beta}-\mathbf{r}_{K}^{\beta}\right)=N z^{2}$. Thus, we see that there are two contributions to the correlator, one dependent on conformations and the other independent.

Eq. (2) formally shows how aspects of interactions $\left(B_{i j}\right)$, conformation space $\left(\mathcal{Q}_{\alpha \beta}\right)$, and sequences $\left(p_{i}\right)$ enter into the nature of statistical dependence. In the following section, we use eq. (2) to demonstrate deviations from REM induced by manipulating each of these three elements.

\section{DEVIATIONS FROM REM}

\section{A. Conformations}

The first term of the energy correlator eq. (2) is proportional to the number of bonds that conformations $\alpha$ and $\beta$ have in common, $\mathcal{Q}_{\alpha \beta}$; clearly, identical bonds give identical contributions to energies of both conformations, thus yielding a dependence. However, if large $\mathcal{Q}_{\alpha \beta}$ are rare, then REM remains a good approximation. To examine this for simple computational models typically employed, we have computed $P(\mathcal{Q})=\sum_{\alpha \beta} \delta\left(\mathcal{Q}_{\alpha \beta}-\mathcal{Q}\right)$ for a variety of conformational spaces.

One type of space consists of maximally compact conformations $(27$-mers and 36 -mers in $3 \times 3 \times 3$ and $3 \times 3 \times 4$ respectively) which are typically used in freezing simulations [5, 10,12]; maximally compact conformations of relatively short chains favor REM validity as small rearrangements are not possible and $P(\mathcal{Q})$ will have virtually no contributions in the large $\mathcal{Q}$ range. In comparison, we considered maximally compact crumpled 64 -mer conformations on the $4 \times 4 \times 4$ lattice. In crumpled conformations, neighbors along the chain are likely to be neighbors in space 13. In this sense, crumpled conformations perhaps crudely model the effect of secondary structure. In our lattice model, the $4 \times 4 \times 4$ cube can be broken down into eight $2 \times 2 \times 2$ subcubes, and crumpled conformations fill every site in a given subcube before entering a new one.

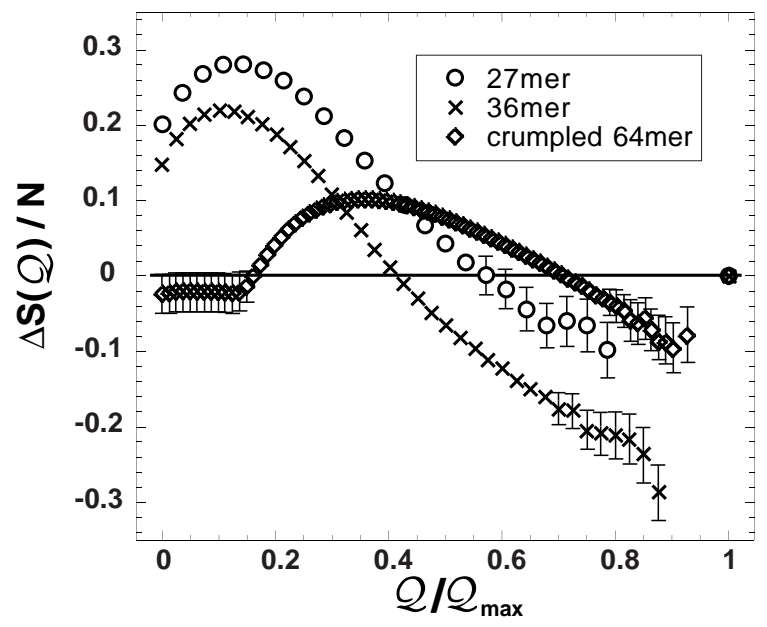

FIG. 1. $\quad \Delta S(\mathcal{Q}) \equiv \ln \left[P(\mathcal{Q}) / P\left(\mathcal{Q}=\mathcal{Q}_{\max }\right)\right]$ for compact 27-mers, compact 36-mers, and compact \& crumpled 64-mers. The discrete region boundary varies greatly: $\mathcal{Q}_{d} / \mathcal{Q}_{\max } \approx 0.6,0.4,0.7$ for 27-mers, 36-mers, and crumpled 64-mers respectively.

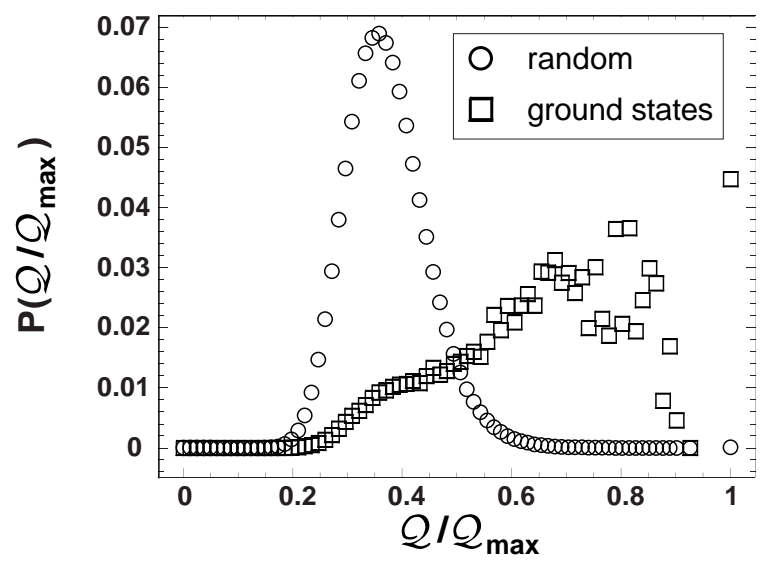

FIG. 2. $P\left(\mathcal{Q} / \mathcal{Q}_{\max }\right)$ for crumpled, compact 64 -mers. We compare the results of conformations taken at random with that obtained by comparing the degenerate ground state conformations of 1000 sequences with Ising interactions. Overlapping ground states signal a departure from REM.

As we see in Fig. 1, $P(\mathcal{Q})$ for all conformational spaces studied are peaked at small $\mathcal{Q}$ (this peak is not at $\mathcal{Q}=0$ due to finite size effects). As $\mathcal{Q}$ increases, $P(\mathcal{Q})$ decreases exponentially, and above a particular $\mathcal{Q}_{d}$, there are at 
most $\mathcal{O}(1)$ conformations available. Thus, for a space with small $\mathcal{Q}_{d}$, there are few states with large overlap and REM is favored. In Fig. 1, we see that crumpled conformations have the greatest $\mathcal{Q}_{d}$ as they allow a greater possibility of rearrangements on small scales (large $\mathcal{Q}$ ).

With the knowledge of the relative favorability of a given conformation space for REM validity, what is the effect of these statistical dependencies on the nature of freezing? While REM formally means that all states are statistically independent, what is relevant is the low energy states. For example, degenerate ground states (as is common in models with "discrete" interactions [14]) will not overlap if they are statistically independent. This holds for 27-mers and 36-mers with Potts interactions $\left(B_{i j}=\delta_{i j}\right)$, whose ground states yield a $P(\mathcal{Q})$ which is indistinguishable from that of conformations taken at random [10,14. In the light of our previous discussion, REM appears to be valid because $\mathcal{Q}_{d}$ is sufficiently small. The situation is different for crumpled 64-mers: upon enumerating [12] the energies of all conformations for 1000 sequences with Ising (2 letter Potts) interactions and comparing the ground states (Fig. 2), we see that the increase in $\mathcal{Q}_{d}$ for crumpled 64-mers is sufficient such that REM fails for this conformational space. This does not merely demonstrate that REM breaks for crumpled 64-mers in particular, but rather REM validity is clearly not an a priori property of all conformation spaces in general, even in three dimensions [3].

\section{B. Interactions}

Interactions enter into the conformational dependent term of eq. (2) through the variance of the elements of the interaction matrix, and thus this term is present for all types of interactions. However, interactions play a more dramatic role in the conformational independent term in eq. (2), as it vanishes for many models, but not, for example, if there is one monomer species that interacts particularly strong with all others; in this case, there is a correlated contribution even when there is no bond in common. The appearance of the conformation independent term signals a departure from REM, as even states with vanishing $\mathcal{Q}$ are statistically dependent.

What forms of interactions have this residual (conformation independent) statistical dependence? One notable example is the HP model $\left(B_{i j}=\delta_{i 1} \delta_{1 j}\right)$ [5]. For even composition overall $\left(p_{i}=1 / q\right)$, but not fixed for a particular sequence, the conformation independent term does not vanish. However, for composition fixed to be even for each sequence and finite chains, there is an additional term to eq. (2) which leads to a negative conformation independent constant. Thus, typical conformations will have a small overlap, but the effect of this overlap is canceled by the negative constant and the result is an effective statistical independence for typical conformations (but anti-dependence (dependence) for conformations with smaller (larger) overlap). Thus, REM may appear to be a good approximation, but only due to a complicated cancellation of factors. Upon examination with eq. (2), the Miyazawa and Jernigan matrix (MJM) 15, a set of amino acid interaction potentials derived from protein statistics, seems like the HP model plus some noise; thus our analysis for HP is applicable to MJM. For models with an even composition and a symmetric contribution from monomer species, such as Potts or Independent Interaction Model (IIM), in which $B_{i j}$ are taken from a Gaussian distribution [3] interactions, the conformation independent term in eq. (2) vanishes and the only statistical dependence comes from conformational overlap.

\section{Sequences}

An uneven composition also leads to the non-vanishing of the conformation independent term (and thus a a statistical dependence) in many models, including Potts and IIM, since there is an imbalance as in the HP model discussed above.

However, while we have been speaking about random sequences, protein sequences are not random. Indeed, theoretically, models of "minimal frustration" [2], "sequence selection" [7], or "imprinting" [8] in which sequences are chosen to have low energies in a desired target conformation $\star$, have been employed as a better model of proteins. If states were truly statistically independent, these procedures would act to "pull down" the energy of the ground state alone; however, statistical dependence between states means that the energy of other states are pulled down as well. Moreover, sequence selection has also been experimentally realized in de novo protein design [9], and REM is an implicit assumption in such experiments: if design leads to many states (not just the desired state $\star$ ) with low energy, this procedure fails.

For our purpose of describing the validity of REM, sequence selection is also a useful tool to demonstrate statistical dependence. In this sense, selection acts as a "field" in which we test the response of the energies of other conformations to the manipulation of the energy of $\star$. In Fig. 3, we see from the density of states, $P(E, \mathcal{Q}) \equiv \sum_{\alpha} \delta\left(\mathcal{Q}-\mathcal{Q}_{\alpha \star}\right) \delta\left(E-\mathcal{H}_{\alpha}\right)$, of a well designed sequence with Ising interactions that sequence selection acts on all states $\alpha$ to a degree that is roughly linear in $\mathcal{Q}_{\alpha \star}$. Therefore, the energy of a particular conformation $\alpha$ is largely determined by $\mathcal{Q}_{\alpha \star}$. Thus, we see that sequence selection does not pull down the energy of a desired conformation but rather affects the whole density of states. This is a dramatic deviation from REM.

However, this effect is not necessarily detrimental. For models with a degree of statistical anti-dependence 
(eg. fixed, even composition HP), selection may act to push up the energy levels of conformations other than $\star$. Moreover, as this statistical anti-dependence varies between sequences, it could be considered as a sequence selection criteria (in additional to optimization of the ground state) and therefore is a possible means to "design out" unwanted conformations [11]. Also, recent models of "protein folding funnels" 5 require conformational space deviations from REM: without some relationship between $\mathcal{Q}$ and energy, there would be no "path" to the ground state and kinetics would be simply a random search through conformation space, requiring the Levinthal time [16].

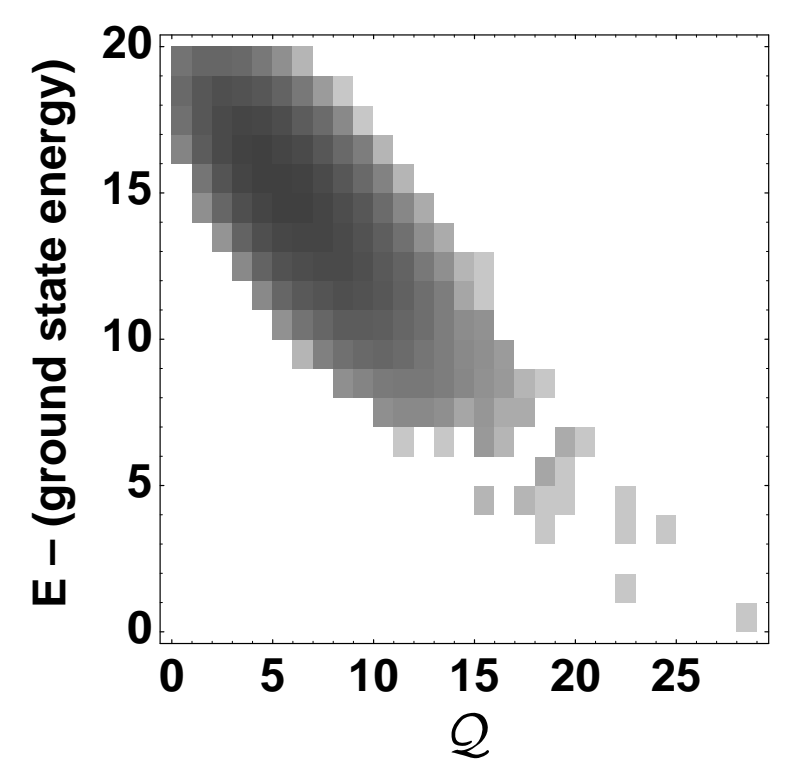

FIG. 3. $P\left(E-E_{\text {gnd }}, \mathcal{Q}\right)$ for maximally compact 27 -mer conformations $\left(\mathcal{Q}_{\max }=28\right)$, Ising interactions $\left(B_{i j}=\delta_{i j}\right)$, and a sequence chosen to have a low ground state energy.

\section{CONCLUSIONS}

While our discussion employs simplified models, such as lattice conformation spaces and a Hamiltonian (1) which includes only pairwise interactions, the aspects which push the system away from REM validity are model independent. Is heteropolymer freezing well described by REM? This complicated question is clearly dependent on all aspects of the system under question. What is clear is that REM validity is not guaranteed for all heteropolymers. For the models commonly employed, it is notable that HP and MJM have extremely questionable REM validity.

Is protein folding well described by REM? From the point of view of eq. (2), interactions between amino acids are in the HP/MJM class; biologically, the possibility of statistical anti-dependence is quite intriguing, and perhaps sheds light on the evolutionary significance of the nature of amino acid interactions. As for the nature of the space of protein conformations, one must consider possible kinetic restrictions as well as the role of secondary structure, which highly restricts the nature of conformational space, perhaps hindering REM. Thus, with possible deviations from REM arising from both interactions and conformational space, REM validity for proteins is questionable.

\section{ACKNOWLEDGEMENTS}

We have benefited from discussions with A. Gutin, M. Kardar, and E. Shakhnovich. The work was supported by NSF (DMR 90-22933) and Project SCOUT (ARPA MDA972-92-J-1032). AYG acknowledges the support of Kao Fellowship.

[1] B. Derrida, Phys. Rev. Lett 45 (1980) 79.

[2] J. D. Bryngelson and P. G. Wolynes, Proc. Nat. Acad. Sci., USA 84 (1987) 7524.

[3] E. Shakhnovich and A. Gutin, Biophys. Chem. 34, 187 (1989); J. Phys. A22, 1647 (1989).

[4] P.G. Wolynes, in Spin Glass Ideas in Biology, edited by D. Stein (World Scientific, Singapore, 1991).

[5] K. Yue and K. A. Dill, Proc. Nat. Acad. Sci., USA 89, 4163 (1992); N. D. Socci and J. N. Onuchic, J. Chem. Phys., 101, 1519 (1994); J. D. Bryngelson, J. N. Onuchic, N. D. Socci, and P. G. Wolynes, Proteins, 21, 167 (1995).

[6] E. I. Shakhnovich and A. M. Gutin, J. Theor. Biol 149, 537 (1991); J. D. Bryngelson, J. Chem. Phys. 100, 6038 (1994); V. S. Pande, A. Yu. Grosberg, and T. Tanaka, J. Chem. Phys. 103, 9482 (1995).

[7] E.I. Shakhnovich and A.M. Gutin, Proc. Nat. Acad. Sci., USA 90, 7195 (1993).

[8] V. S. Pande, A. Yu. Grosberg, and T. Tanaka, Macromolecules 28, 2218 (1995).

[9] M. H. Hecht, J. S. Richardson, and D. C. Richardson, Science 249, 884 (1990).

[10] E. Shakhnovich, and A. Gutin, J Chem. Phys., 93, 5967 (1990).

[11] K. Yue, K. M. Fiebig, P. D. Thomas, H. S. Chan, K. A. Dill, and E. I. Shakhnovich, Proc. Nat. Acad. Sci, USA 92, 325 (1995); H. Li, R. Helling, C. Tang, and N. Wingreen, cond-mat preprint 9603016 (1996).

[12] V. S. Pande, C. Joerg, A. Yu. Grosberg, and T. Tanaka, J. Phys. A27, 6231 (1994).

[13] A. Yu. Grosberg, S. K. Nechaev, and E. I. Shakhnovich, J. de Phys. (France) 49, 2095 (1988).

[14] A. M. Gutin and E. I. Shakhnovich, J Chem. Phys., 98, 8174 (1993).

[15] S. Miyazawa and R. Jernigan, Macromolecules 18, 534 (1985).

[16] J. D. Bryngelson and P. G. Wolynes, J. of Phys. Chem. 936902 (1989). 